\title{
Supplemental Material
}

\section{Immunotoxic potentials of bisphenol F mediated through lipid}

\section{signaling pathways on macrophages}

Chao Zhao, ${ }^{\dagger}$ Zhi Tang, ${ }^{\dagger}$ Peisi Xie, ${ }^{\dagger}$ Kaili Lin, ${ }^{\S}$ Arthur Chi Kong Chung, ${ }^{\dagger}$ and Zongwei Cai* ${ }^{\dagger}$

†State Key Laboratory of Environmental and Biological Analysis, Department of

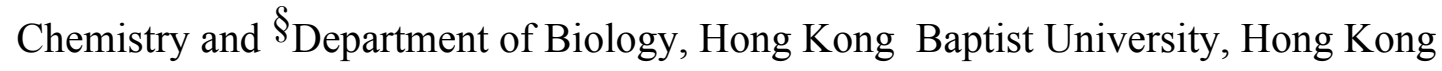
SAR 999077, China

\$Shenzhen Center for Disease Control and Prevention, Shenzhen 518055, China

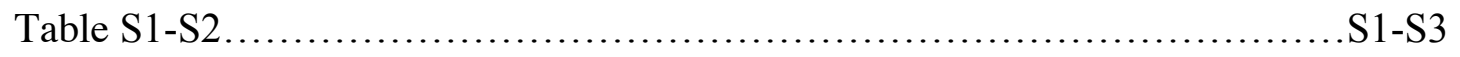

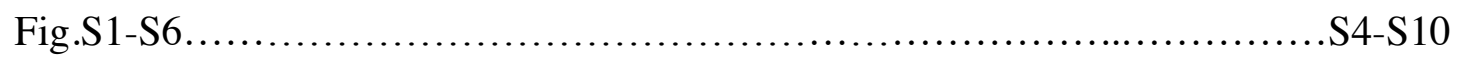


Table S1. Differential lipid metabolites induced by BPF exposure in macrophages

\begin{tabular}{|c|c|c|c|c|c|}
\hline & Lipid & Composition & $\mathbf{m} / \mathbf{z}$ & Formula & $\begin{array}{c}\text { Errors } \\
\text { (ppm) }\end{array}$ \\
\hline \multirow{8}{*}{ SM } & SM d 40:1 & $\mathrm{SM}(\mathrm{d} 22: 0 / 18: 1)$ & 786.6615 & C45 H91 O6 N2 P1 & 1 \\
\hline & SM d40:2 & $\mathrm{SM}(\mathrm{d} 22: 1 / 18: 1)$ & 784.6458 & C45 H89 O6 N2 P1 & 1 \\
\hline & SM d42:1 & $\mathrm{SM}(\mathrm{d} 14: 0 / 28: 1)$ & 814.6928 & C47 H95 O6 N2 P1 & 1 \\
\hline & SM d42:1 & $\mathrm{SM}(\mathrm{d} 24: 0 / 18: 1)$ & 814.6928 & C47 H95 O6 N2 P1 & 1 \\
\hline & SM d 42:2 & $\mathrm{SM}(\mathrm{d} 18: 1 / 24: 1)$ & 812.6771 & C47 H93 O6 N2 P1 & 1 \\
\hline & SM d42:4 & $\operatorname{SM}(\mathrm{d} 22: 0 / 20: 4)$ & 808.6512 & C47 H89 O6 N2 P1 & 1 \\
\hline & SM d43:1 & $\operatorname{SM}(\mathrm{d} 25: 0 / 18: 1)$ & 828.7084 & C48 H97 O6 N2 P1 & 1 \\
\hline & SM d43:2 & $\operatorname{SM}(\mathrm{d} 18: 1 / 25: 1)$ & 826.6928 & C48 H95 O6 N2 P1 & 1 \\
\hline \multirow{7}{*}{ Cer } & Cer d34:2 & $\operatorname{Cer}(\mathrm{d} 18: 2 / 16: 0)$ & 535.4964 & C34 H65 O3 N1 & 1 \\
\hline & Cer d40:2 & $\operatorname{Cer}(\mathrm{d} 18: 1 / 22: 1)$ & 619.5903 & C40 H77 O3 N1 & 1 \\
\hline & Cer d41:1 & $\operatorname{Cer}(\mathrm{d} 17: 1 / 24: 0)$ & 635.6216 & C41 H81 O3 N1 & 1 \\
\hline & Cer d42:1 & Cer(d18:1/24:0) & 649.6373 & C42 H83 O3 N1 & 1 \\
\hline & Cer d42:2 & $\operatorname{Cer}(\mathrm{d} 18: 1 / 24: 1)$ & 647.6216 & C42 H81 O3 N1 & 1 \\
\hline & Cer $\mathrm{d} 42: 3$ & $\operatorname{Cer}(\mathrm{d} 18: 2 / 24: 1)$ & 645.6063 & C42 H79 O3 N1 & 0 \\
\hline & Cer d44:2 & $\operatorname{Cer}(\mathrm{d} 18: 1 / 26: 1)$ & 675.6529 & C44 H85 O3 N1 & 0 \\
\hline \multirow{2}{*}{ So } & So $18: 1$ & So $(\mathrm{d} 18: 1)$ & 299.2824 & $\mathrm{C} 18 \mathrm{H} 38 \mathrm{O} 2 \mathrm{~N} 1$ & 1 \\
\hline & So $18: 0$ & So (d18:0) & 301.2981 & $\mathrm{C} 18 \mathrm{H} 40 \mathrm{O} 2 \mathrm{~N} 1$ & 1 \\
\hline \multirow{20}{*}{$\mathrm{PC}$} & PC 26:0 & $\operatorname{PC}(12: 0 / 14: 0)$ & 649.4683 & C34 H68 O8 N1 P1 & 0 \\
\hline & PC $31: 1$ & $\operatorname{PC}(17: 1 / 14: 0)$ & 717.5309 & C39 H76 O8 N1 P1 & 0 \\
\hline & PC 32:1 & $\operatorname{PC}(16: 0 / 16: 1)$ & 731.5465 & C40 H78 O8 N1 P1 & 0 \\
\hline & PC 35:1 & $\operatorname{PC}(19: 1 / 16: 0)$ & 773.5935 & C43 H84 O8 N1 P1 & 0 \\
\hline & PC 35:1 & $\operatorname{PC}(17: 0 / 18: 1)$ & 773.5935 & C43 H84 O8 N1 P1 & 0 \\
\hline & PC 35:3 & $\mathrm{PC}(18: 0 / 17: 3)$ & 769.5622 & C43 H80 O8 N1 P1 & 1 \\
\hline & PC $36: 2$ & $\operatorname{PC}(18: 1 / 18: 1)$ & 785.5935 & C44 H84 O8 N1 P1 & 1 \\
\hline & PC 42:10 & $\operatorname{PC}(20: 4 / 22: 6)$ & 853.5622 & C50 H80 O8 N1 P1 & 1 \\
\hline & PC 44:2 & $\mathrm{PC}(26: 1 / 18: 1)$ & 897.7187 & C52 H100 O8 N1 P1 & 1 \\
\hline & PC O-32:1 & $\mathrm{PC}(16: 0 \mathrm{e} / 16: 1)$ & 717.5672 & C40 H80 O7 N1 P1 & 1 \\
\hline & PC O-33:1 & $\mathrm{PC}(16: 0 \mathrm{e} / 17: 1)$ & 731.5829 & C41 H82 O7 N1 P1 & 1 \\
\hline & PC O-34:2 & $\mathrm{PC}(16: 0 \mathrm{e} / 18: 2)$ & 743.5829 & C42 H82 O7 N1 P1 & 1 \\
\hline & PC O-34:4 & $\mathrm{PC}(14: 0 \mathrm{e} / 20: 4)$ & 739.5516 & C42 H78 O7 N1 P1 & 0 \\
\hline & PC O-38:5 & $\mathrm{PC}(16: 0 \mathrm{e} / 22: 5)$ & 793.5985 & C46 H84 O7 N1 P1 & 0 \\
\hline & PC O-42:6 & $\mathrm{PC}(20: 0 \mathrm{e} / 22: 6)$ & 847.6455 & C50 H90 O7 N1 P1 & 0 \\
\hline & PC P-32:1 & $\mathrm{PC}(16: 0 \mathrm{p} / 16: 1)$ & 715.5516 & C40 H78 O7 N1 P1 & 0 \\
\hline & PC P-34:0 & $\mathrm{PC}(16: 0 \mathrm{p} / 18: 0)$ & 745.5985 & C42 H84 O7 N1 P1 & 0 \\
\hline & PC P-38:3 & $\mathrm{PC}(18: 0 \mathrm{p} / 20: 3)$ & 795.6142 & C46 H86 O7 N1 P1 & 1 \\
\hline & PC P-40:4 & $\mathrm{PC}(18: 0 \mathrm{p} / 22: 4)$ & 821.6298 & C48 H88 O7 N1 P1 & 0 \\
\hline & PC P-40:5 & $\mathrm{PC}(18: 0 \mathrm{p} / 22: 5)$ & 819.6142 & C48 H86 O7 N1 P1 & 0 \\
\hline \multirow{7}{*}{$\mathrm{PE}$} & PE 36:2 & $\operatorname{PE}(18: 1 / 18: 1)$ & 743.5465 & C41 H78 O8 N1 P1 & 1 \\
\hline & PE 36:3 & $\operatorname{PE}(18: 1 / 18: 2)$ & 741.5309 & C41 H76 O8 N1 P1 & 1 \\
\hline & PE 36:4 & $\operatorname{PE}(16: 0 / 20: 4)$ & 739.5152 & C41 H74 O8 N1 P1 & 1 \\
\hline & PE 36:5 & $\operatorname{PE}(16: 0 / 20: 5)$ & 737.4996 & C41 H72 O8 N1 P1 & 0 \\
\hline & PE 38:1 & $\operatorname{PE}(18: 0 / 20: 1)$ & 773.5935 & C43 H84 O8 N1 P1 & 0 \\
\hline & PE 38:3 & $\operatorname{PE}(18: 0 / 20: 3)$ & 769.5622 & C43 H80 O8 N1 P1 & 0 \\
\hline & PE 40:5 & $\operatorname{PE}(20: 1 / 20: 4)$ & 793.5689 & C45 H80 O8 N1 P1 & 0 \\
\hline
\end{tabular}




\begin{tabular}{|c|c|c|c|c|c|}
\hline \multirow{4}{*}{} & PE 40:6 & PE(18:1/22:5) & 791.5465 & C45 H78 O8 N1 P1 & 0 \\
\cline { 2 - 6 } & PE P-40:2 & PE(18:1p/22:1) & 783.6142 & C45 H86 O7 N1 P1 & 0 \\
\hline \multirow{3}{*}{ PS } & PS 36:1 & PS (18:0/18:1) & 789.5520 & C42 H80 O10 N1 P1 & 0 \\
\cline { 2 - 6 } & PS 36:2 & PS (18:1/18:1) & 787.5363 & C42 H78 O10 N1 P1 & 0 \\
\cline { 2 - 6 } & PS 40:6 & PS(18:0/22:6) & 835.5343 & C46 H78 O10 N1 P1 & 0 \\
\hline
\end{tabular}


Table S2. Effects of BPF on lipidomics-related gene expression in $10^{-10} \mathrm{M}$ and $10^{-8} \mathrm{M}$ BPF-treated macrophages

\begin{tabular}{|c|c|c|c|c|c|}
\hline \multirow{3}{*}{$\begin{array}{c}\text { Lipid Metabolic } \\
\text { Pathways }\end{array}$} & \multirow{3}{*}{ Gene } & \multicolumn{4}{|c|}{ Gene Expression Fold } \\
\hline & & \multicolumn{2}{|c|}{$\begin{array}{c}10^{-10} \mathrm{M} \text { of } \mathrm{BPF} / \\
\text { Control }\end{array}$} & \multicolumn{2}{|c|}{$\begin{array}{c}10^{-8} \mathrm{M} \text { of } \mathrm{BPF} / \\
\text { Control }\end{array}$} \\
\hline & & $\mathbf{P}$ & Fold & $\mathbf{P}$ & Fold \\
\hline \multirow{3}{*}{$\begin{array}{c}\text { (1). Cer De novo synthetic } \\
\text { pathway }\end{array}$} & Sptlc & 0.05 & 1.6 & 0.03 & 2.3 \\
\hline & CerS & 0.04 & 1.6 & 0.03 & 3.0 \\
\hline & SphK2 & 0.10 & 1.2 & 0.10 & 4.3 \\
\hline (2). SM hydrolysis & SMase & 0.03 & 2.3 & 0.11 & 2.9 \\
\hline \multirow{2}{*}{$\begin{array}{c}\text { (3). Cer degradation } \\
\text { pathway }\end{array}$} & CDase & 0.02 & 1.3 & 0.12 & 2.7 \\
\hline & S1P lyase & 0.32 & 2.6 & 0.05 & 3.9 \\
\hline \multirow{3}{*}{ (4).Kennedy pathway } & Ccpt & 0.11 & 2.2 & 0.03 & 2.0 \\
\hline & Pcyt2 & 0.02 & 3.4 & 0.11 & 3.5 \\
\hline & Cept & 0.20 & -0.8 & 0.04 & -0.5 \\
\hline $\begin{array}{c}\text { (5). PS Decarboxylation } \\
\text { Pathway }\end{array}$ & Psd & 0.05 & -0.4 & 0.03 & -0.6 \\
\hline $\begin{array}{l}\text { (6). PEMT methylation } \\
\text { pathway }\end{array}$ & Pemt & 0.04 & 1.3 & 0.02 & 2.0 \\
\hline \multirow{2}{*}{ (7). PS synthesis } & Pss 1 & 0.03 & -0.5 & 0.02 & 0.9 \\
\hline & Pss2 & 0.04 & -0.4 & 0.24 & -0.6 \\
\hline
\end{tabular}


Figure S1. Apoptosis-related gene expression in macrophages following BPF and BPA exposure. Data were analyzed as means \pm SD of eight replicate experiments (the symbol represents statistical significance between treated group and control group: * $\mathrm{P}<0.05, * * \mathrm{P}<0.01$; the same as below).
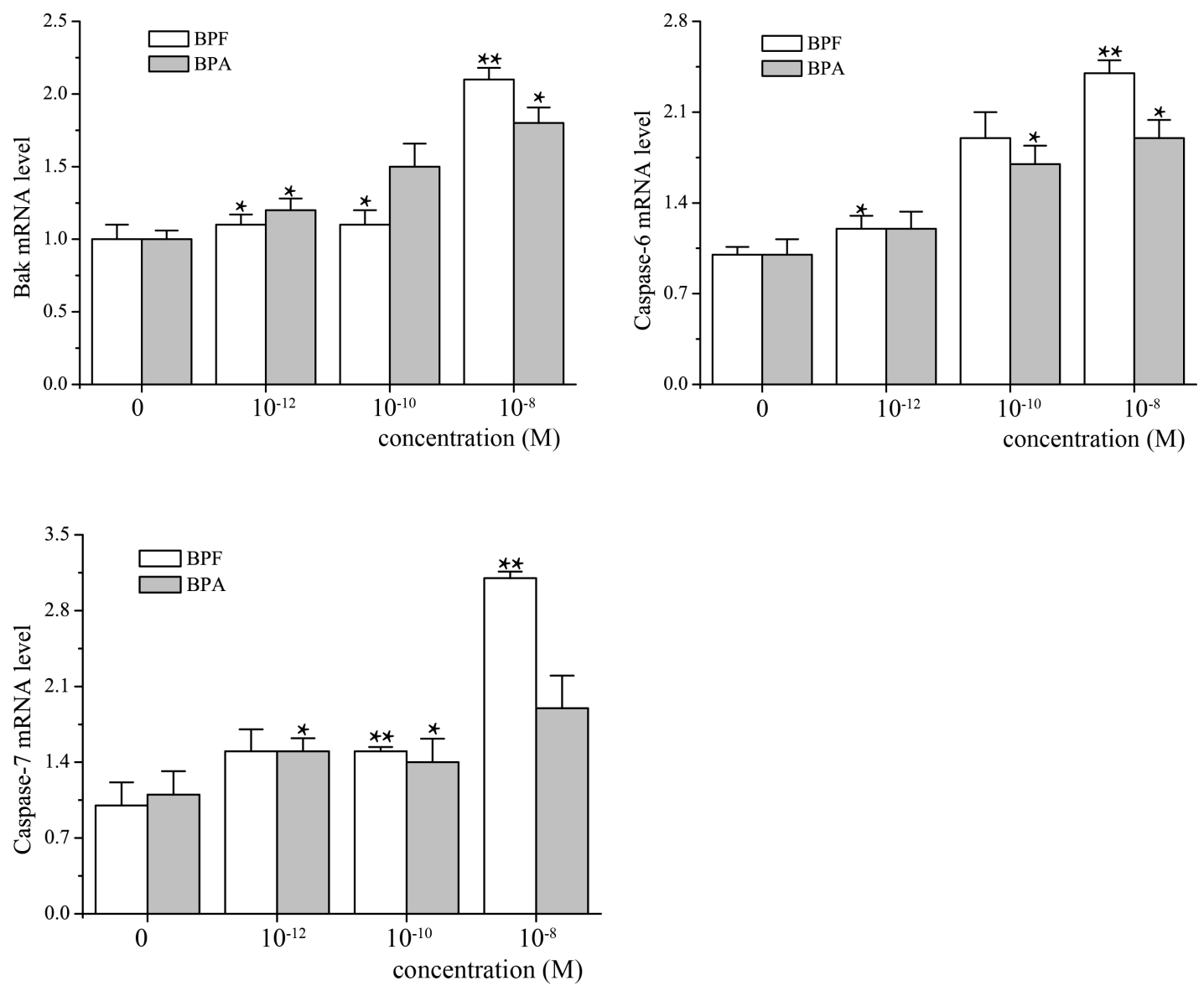
Figure S2. (A) Effects of BPA on macrophage apoptosis. Macrophages were administrated with BPF for $24 \mathrm{~h}$, and Annexin V-FITC/PI was used to determine the cell apoptosis via flow cytometry. (B) The percentages of apoptotic cells were calculated from the ratio of apoptotic cells to total cells counted.

(A)

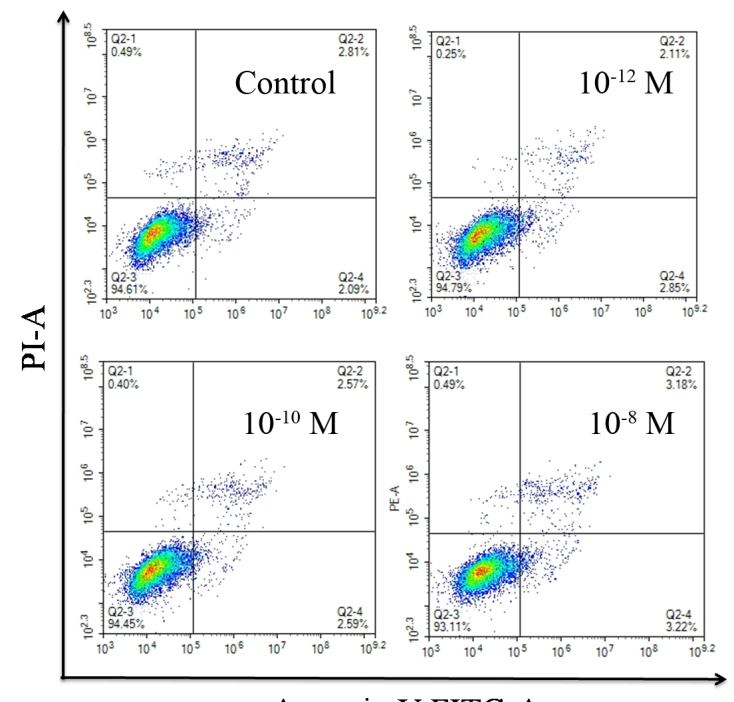

Annexin V-FITC-A

(B)
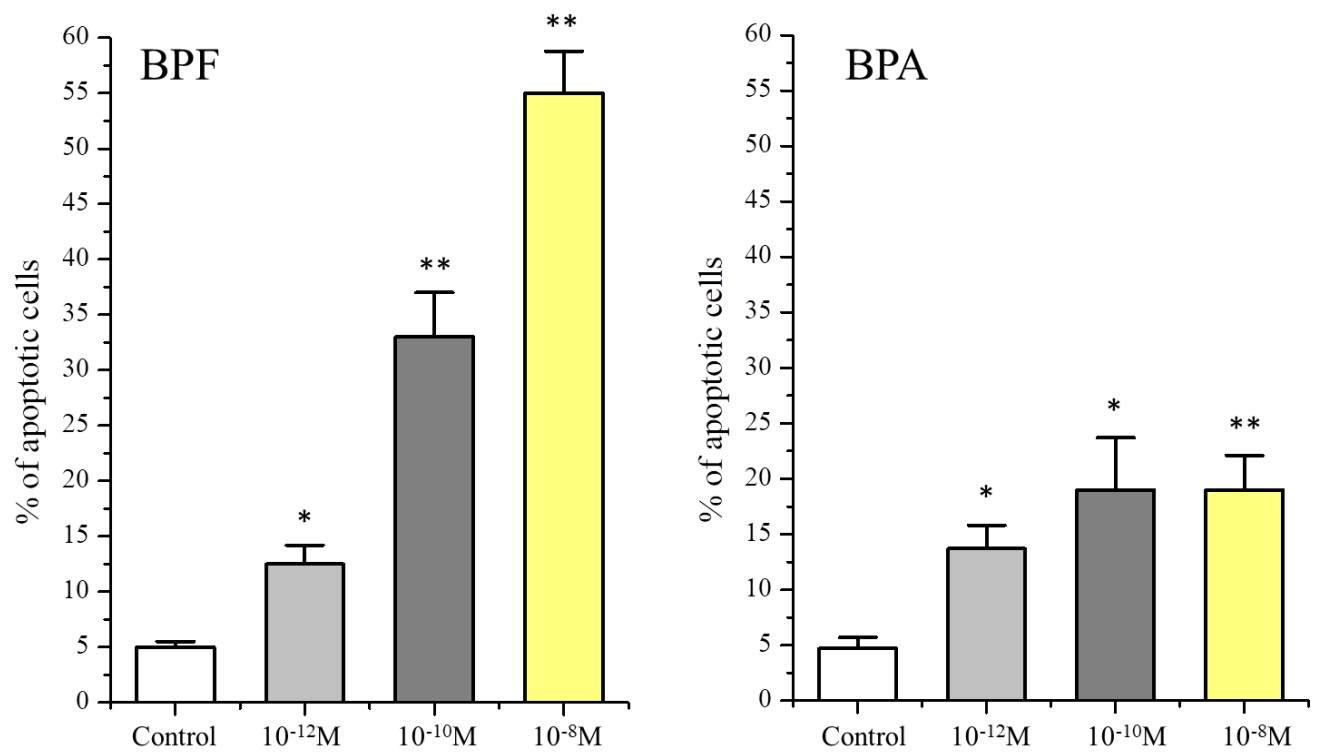
Figure S3. ROS formation in macrophages following BPF and BPA exposure. (A) MDA level; (B) GSH-Px activities; (C) SOD activities; (D) Effect of NAC on ROS generation. Macrophages were treated to NAC for $1 \mathrm{~h}$ firstly, and then co-incubated with $10^{-8} \mathrm{M}$ of BPF or BPA.

(A)

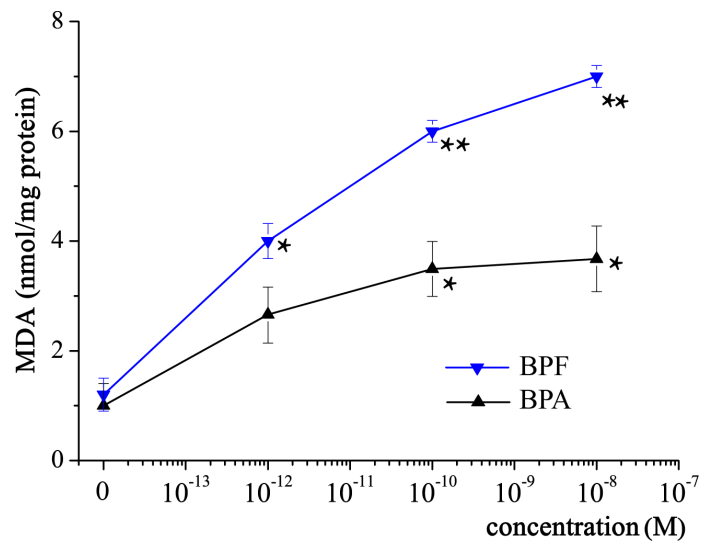

(C)

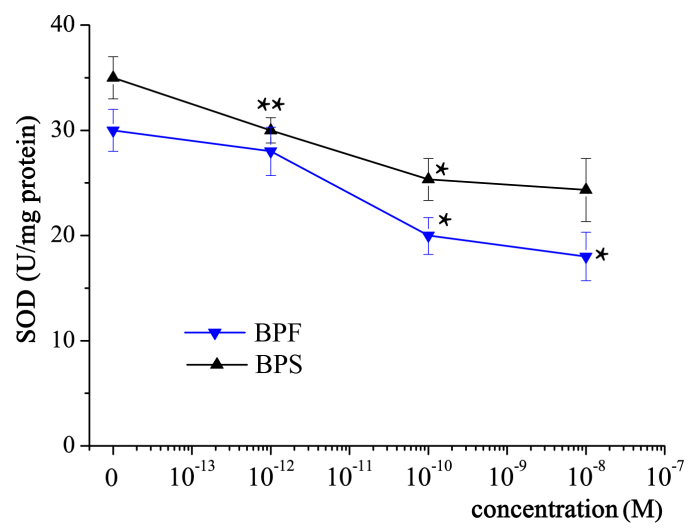

(B)

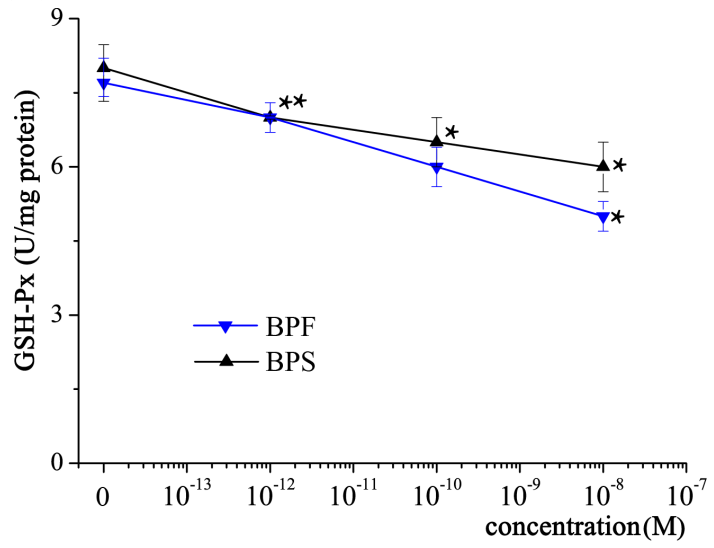

(D)

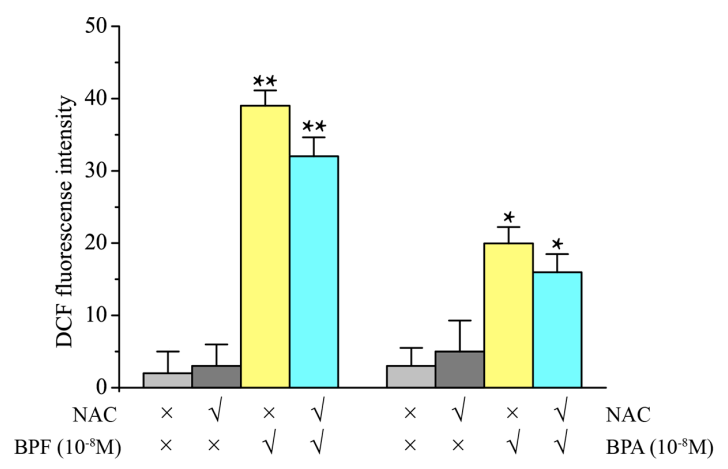


Figure S4. Expression and secretion of immune-related cytokines in macrophages (TGF- $\beta$ and IL-10) following BPF (A and B) and BPA exposure (C and D).

(A)

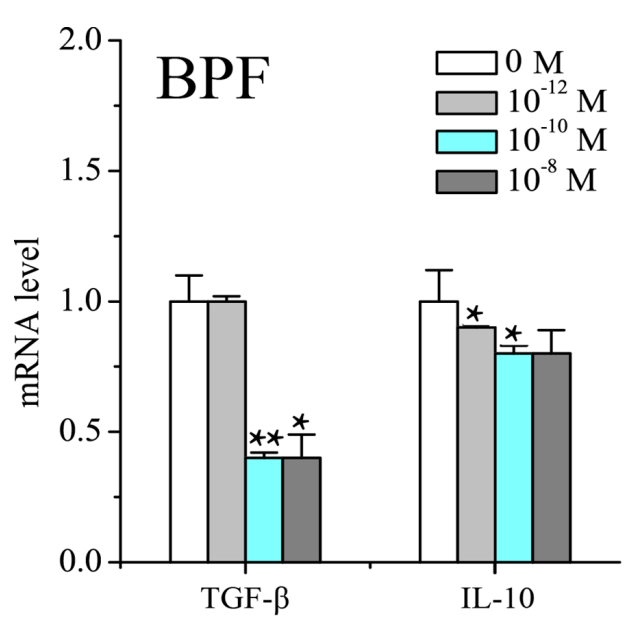

(C)

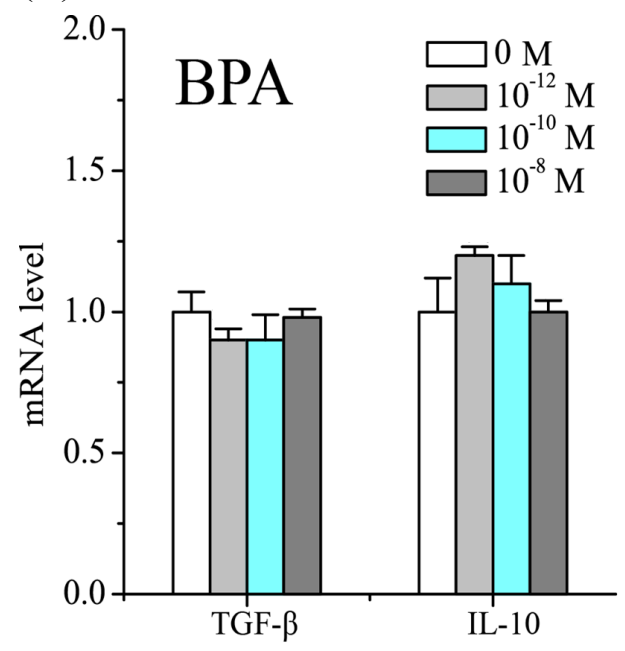

(B)

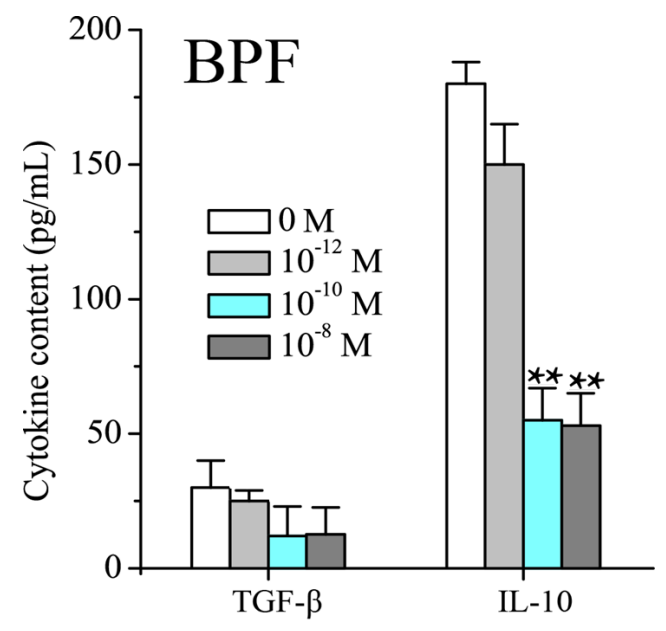

(D)

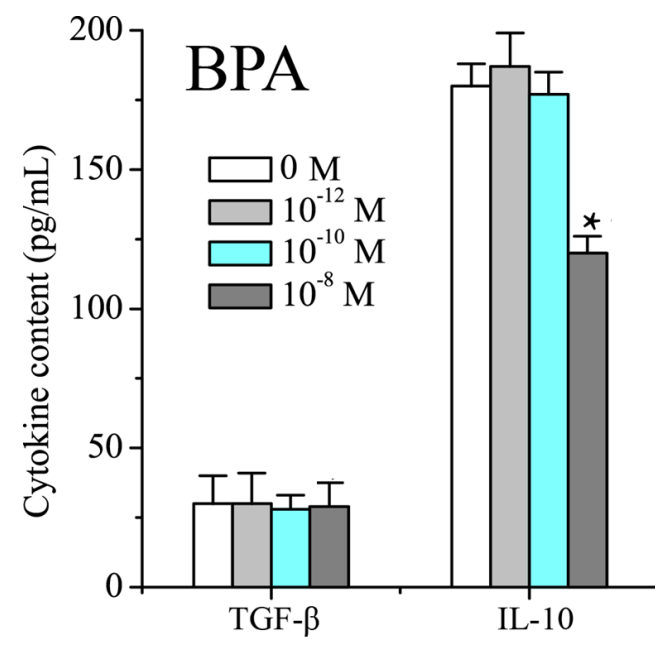


Figure S5. Lipidomics analysis in macrophages following BPF exposure. The PLS-DA score plots in positive (A) and negative ionization modes (B). The doses of $10^{-8}, 10^{-10}$ and $10^{-12} \mathrm{M} \mathrm{BPF}$, negative control (0.002\% DMSO in culture medium) and positive control (M1 phenotype) were used to multivariate statistical analysis $(n=8)$.
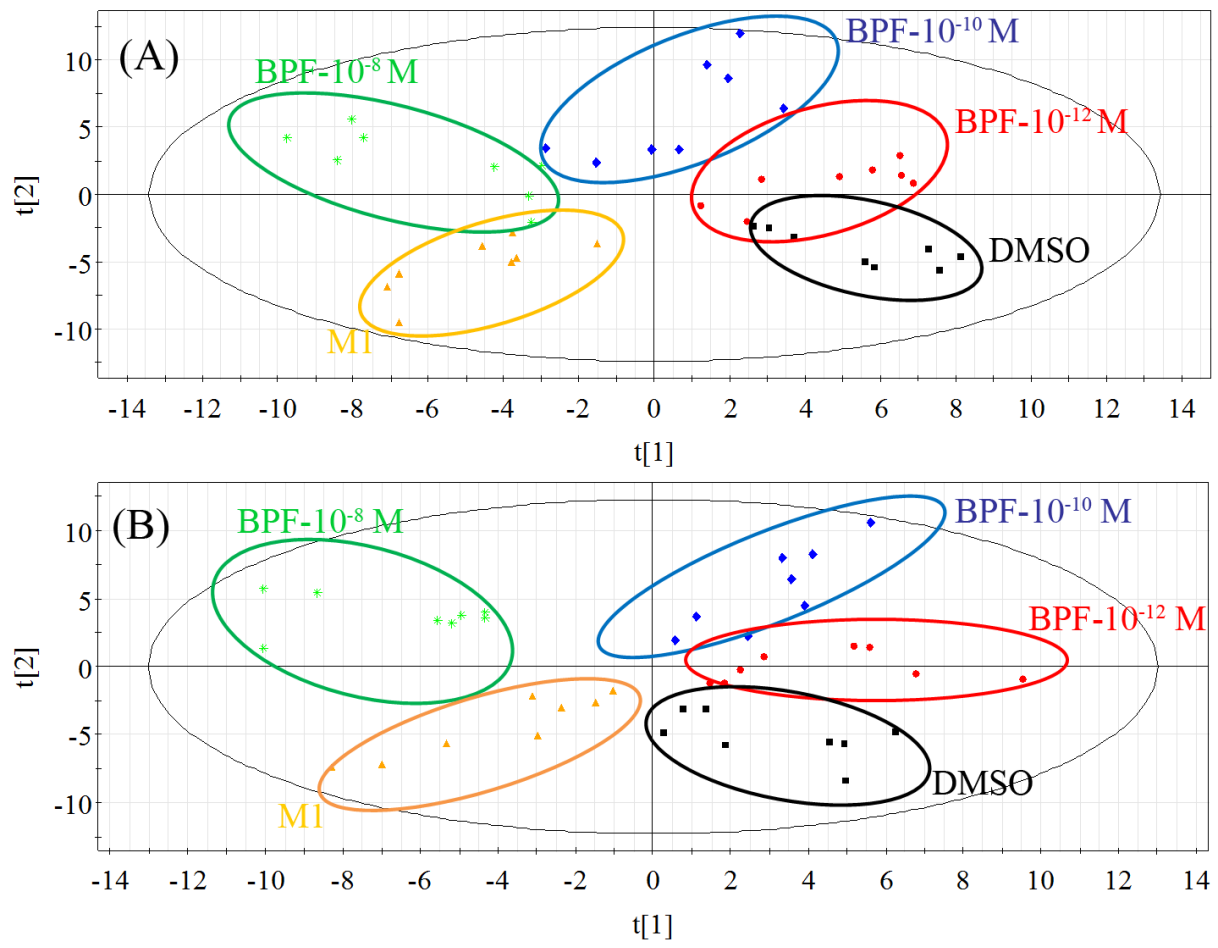
Figure S6. (A) Composition of acyl chains in four GPs categories including PC, PE, PI and PG. The identified chain composition of lipids by software LipidSearch were used the calculation of percentage of ether-linked FA, PUFA, MUFA- and SFA. (B) Heat map analysis for identified subclass lipids. The individual samples were represented in the vertical axis, and the identified lipids were represented in the horizontal axis. Up-, un- and down-regulated lipids were represented in red, black and green, respectively.

(A)

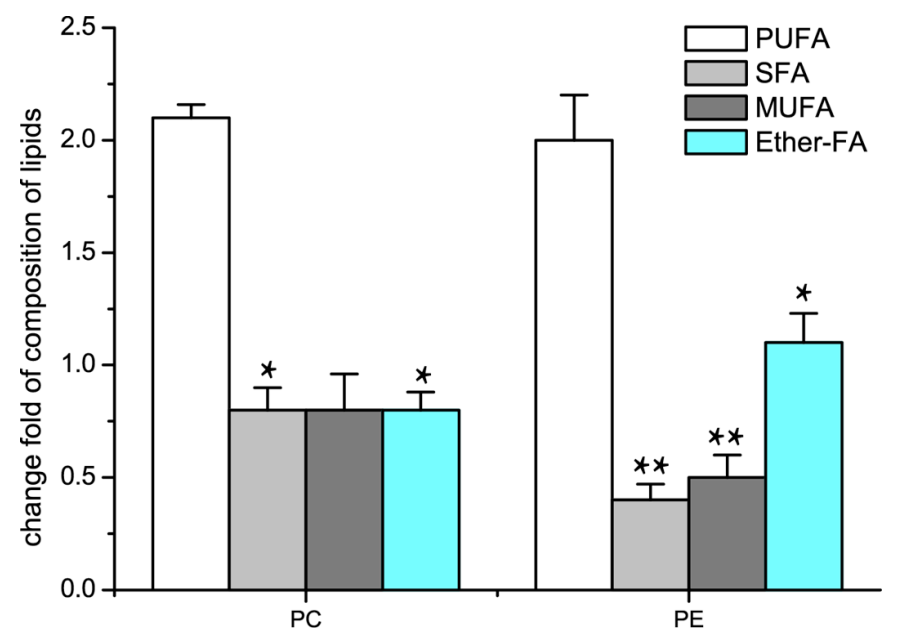

(B) 

Control
$10^{-12} \mathrm{M}$
$10^{-10} \mathrm{M}$
$10^{-8} \mathrm{M}$
M1

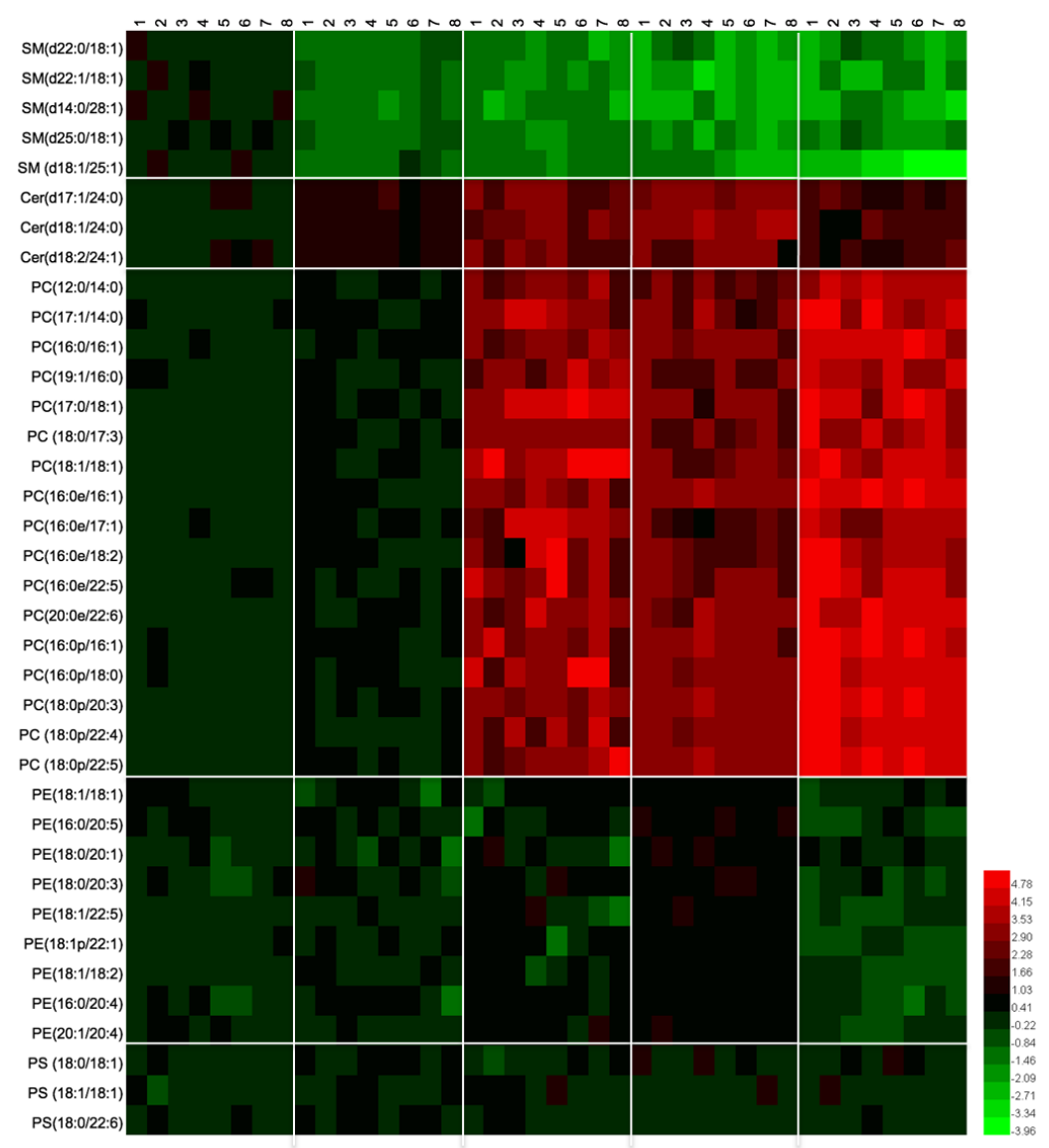

